\title{
Trends Cycles and Seasons: Econometric Methods of Signal Extraction
}

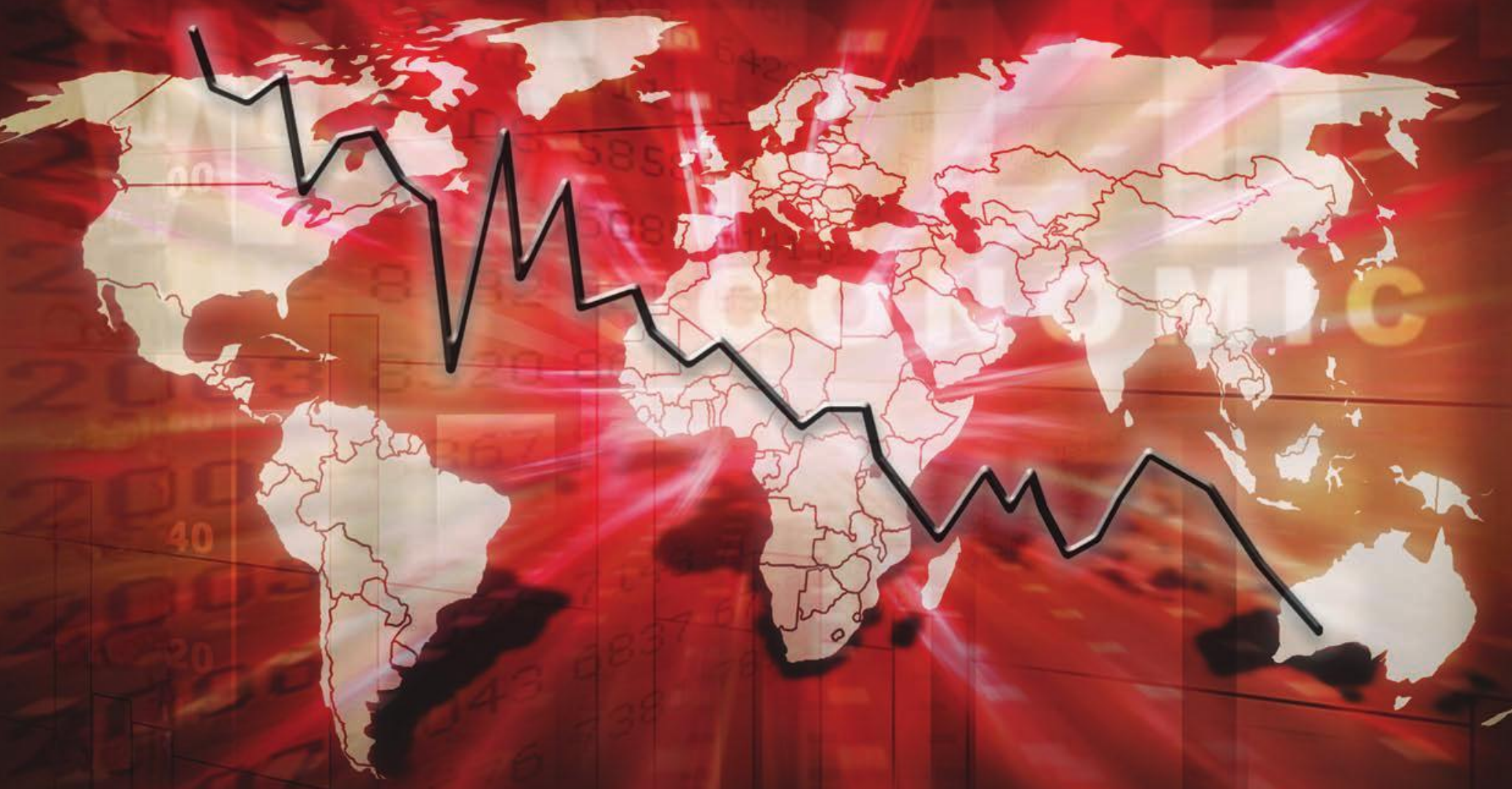

Stephen Pollock, University of Leicester, UK

Working Paper No. 14/04

February 2014 


\title{
TRENDS CYCLES AND SEASONS: ECONOMETRIC METHODS OF SIGNAL EXTRACTION
}

\author{
By D.S.G. POLLOCK \\ University of Leicester \\ Email: stephen_pollock@sigmapi.u-net.com
}

\begin{abstract}
Alternative methods of trend extraction and of seasonal adjustment are described that operate in the time domain and in the frequency domain. The time-domain methods that are implemented in the TRAMO-SEATS and the STAMP programs are described and compared. An abbreviated time-domain method of seasonal adjustment that is implemented in the IDEOLOG program is also described. Finite-sample versions of the Wiener-Kolmogorov filter are described that can be used to implement the methods in a common way.

The frequency-domain method, which is also implemented in the IDEOLOG program, employs a ideal frequency selective filter that depends on identifying the ordinates of the Fourier transform of a detrended data sequence that should lie in the pass band of the filter and those that should lie in its stop band. Filters of this nature can be used both for extracting a low-frequency cyclical component of the data and for extracting the seasonal component.
\end{abstract}

\section{Introduction}

The econometric methods of signal extraction have attained a high degree of sophistication. The X-11 method is still the prevalent one. The X-11 program was the culmination of the pioneering work undertaken by Julius Shiskin in the U.S. Bureau of Census in the 1950's and 1960's, and it was described by Shiskin et al. (1967).

The X-11 program has undergone numerous improvements and modifications, leading to the X-11-ARIMA software packages of 1975 and 1988. (See Dagum 1980, 1988.) Its latest incarnation is in the X-12-ARIMA package. (See Findlay et al. 1998) Much of the relevant information on the method has been provided in a monograph of Ladiray and Quenneville (2001).

Recently, there has been a shrift away from the X-11 method toward modelbased procedures. The dominant model-based method is based on the model of Box and Jenkins (1976) of airline passenger data. It is implemented in the TRAMOSEATS program of Maravall and others. (see Caporello and Maravall 2004)

Another model-based procedure that has become increasingly current, albeit to a lesser extent, is the structural approach of STAMP. (See Koopman et al. 2000) There are also indications that the Census Bureau itself is becoming more eclectic in its approach to seasonal adjustment. (See Monsell, Aston and Koopman 2003 and Monsell 2009)

Both of the above-mentioned model-based procedures depend on the fitting of ARIMA models to the data. The requirement to estimate the models imposes restrictions on their structures, which render them identifiable. The two procedures secure the identifiably of their models in different ways. 


\section{D.S.G. POLLOCK: Econometric Signal Extraction}

In some contexts, it may be appropriate to forego the estimation of a model; and we shall outline one method that does so. This method, which may also be described as a model-based procedure, has been implemented in the computer program IDEOLOG, which is available, together with its code in Pascal, at the address

$$
\text { http://www.le.ac.uk/users/dsgp1/ }
$$

It must be said that this program contains none of the facilities that are available in TRAMO-SEATS and STAMP for dealing with the various data anomalies that are liable to afflict the business of seasonal adjustment.

One of the purposes of this paper is to compare the features of these modelbased approaches and to describe a common way in which they can be implemented that differs from the contrasting ways followed by TRAMO-SEATS and STAMP.

Another purpose of the paper is to provide an alternative method of decomposition that does not depend on the specification an ARIMA model. This is a frequency-domain method that is based on concepts of Fourier analysis. It possesses of certain flexibilities that enable it to serve different purposes from those of the aforementioned ARIMA procedures.

In particular, the frequency-domain method facilitates the isolation of components that are band-limited in frequency and which thereby constitute analytic functions. In the context of business cycle analysis, this will enable one to find the turning points very easily by the methods of differential calculus

In order to achieve a viable exposition of these various methods, we shall need to punctuate the account by descriptions of the underlying mathematical methods. This will give rise, unavoidably, to a somewhat disjunct account.

We begin with of the essential features of the Wiener-Kolmogorov method of signal extraction, which is common to the ARIMA-based methods of signal extraction and to which the frequency-domain method can also be assimilated, as has been shown by Pollock (2007).

\section{Mathematical Difficulties}

For the most part, the theory of signal extraction, as expounded by electrical engineers, presupposes signals of finite duration and of finite energy, which means that they are square integrable. The sampled signals are therefore assumed to be absolutely summable and they are amenable to the $z$-transform within a welldefined region of convergence. Such signals are also amenable to an ordinary Fourier analysis.

The preponderant methods of statistical time series analysis cater to discretetime signals that are statistically stationary. The signals are typically defined over a doubly-infinite index set, and, being of infinite energy, they are not summable. Therefore, the emphasis is shifted from the signals to their autocorrelation functions, which, according the assumptions of ergodicity, are absolutely summable.

In particular, the conditions that guarantee the stability of an autoregressive moving-average process are sufficient to ensure that the $z$-transform of its autocovariance function will converge everywhere on and inside the unit circle. The spectral density function of the process is generated as the complex argument $z$ travels around the circumference of the unit circle. Moreover, although stationary stochastic signals are not amenable to an ordinary Fourier analysis, they are 


\section{D.S.G. POLLOCK: Econometric Signal Extraction}

amenable to a generalised harmonic analysis of the sort that was originally propounded by Wiener (1930).

In econometric applications, the data sequences commonly exhibit strong trends. Therefore, they are not directly amenable to the analysis of doubly-infinite stationary processes. A common recourse has been to reduce the data to stationarity by taking their differences and to proceed with the analysis of the stationary, differenced sequences.

When parametric models are required of trending data, these are liable to be linear stochastic models of the ARIMA variety incorporating roots of unit modulus within the autoregressive operators, which will be removed by the process of differencing.

However, the unit roots within the models pose certain unavoidable mathematical difficulties that are fairly described as embarrassments. These inhibit the free use of the ordinary polynomial algebra in manipulating the equations of such models. The problems can be illustrated by simple first-order difference operator, which is the polynomial $\nabla(z)=1-z$ that has a single real-valued root of unity.

This operator would be effective in reducing a first-order random walk $y(t)=$ $\left\{y_{t}=y_{t-1}+\varepsilon_{t} ; t=0,1,2, \ldots\right\}$, defined on the non-negative integers, to a white noise sequence $\varepsilon(t)=\left\{\varepsilon_{t}=y_{t}-y_{t-1} ; t=1,2, \ldots\right\}$. Thus, on defining $y(z)=$ $\left\{y_{0}+y_{1} z+y_{2} z^{2}+\cdots\right\}$ and $\varepsilon(z)=\left\{\varepsilon_{0}+\varepsilon_{1} z+\varepsilon_{2} z^{2}+\cdots\right\}$ and on adopting the conventions that $y_{-1}=0$ and that $y_{0}=\varepsilon_{0}$, we have

$$
(1-z) y(z)=\varepsilon(z) \quad \text { and, conversely, } \quad y(z)=(1-z)^{-1} \varepsilon(z) .
$$

Provided that $z$ lies within the unit circle, even within an epsilon of its circumference, then these various series will converge. However, if $y(z)=\left\{y_{t} ; t=\right.$ $0, \pm 1, \pm 2, \ldots\}$ and $\varepsilon(z)=\left\{\varepsilon_{t} ; t=0, \pm 1, \pm 2, \ldots\right\}$ are stationary processes defined on a doubly-infinite index, then none of the series will converge.

There is no wholly satisfactory resolution for these problems; and one is liable to take a mixed approach that obeys the necessary restrictions in some contexts and that disregards then in others. The easements that are afforded by ignoring the non-stationarity implied by the presence of autoregressive unit roots are at the cost of having to accept improper rational transfer functions, for which there are no convergent power series expansions, and pseudo spectral density functions that are unbounded at certain frequencies. However, although such an approach is liable to mislead an incautious analyst, the insights that are afforded by a spectral analysis, albeit an improper one, cannot be willingly foregone.

In the section that follows, we shall employ a matrix formulation to accommodate the case of a trended sequence of a finite duration, and, in subsequent sections, we shall revert to the (improper) $z$-transform notation.

\section{Lower-Triangular Toeplitz Matrices}

The algebra of the conventional econometric approach to ARIMA-based signal extraction can be expressed in terms of lower triangular $(L-T)$ Toeplitz matrices. Such matrices are closely related to ordinary algebraic polynomials with which they share the essential property of commutativity. The matrices are of full rank.

Consider the polynomial

$$
\alpha(z)=\alpha_{0}+\alpha_{1} z+\cdots+\alpha_{p-1} z^{p-1}+\alpha_{p} z^{p}
$$




\section{D.S.G. POLLOCK: Econometric Signal Extraction}

of degree $p$, and let $L_{T}=\left[e_{1}, e_{2}, \ldots, e_{T-1}, 0\right]$ be the matrix lag operator of order $T$ derived from the identity matrix $I_{T}=\left[e_{0}, e_{1}, e_{2}, \ldots, e_{T-1}\right]$ by deleting the leading column and by adding a column of zeros to the end of the array. Then, the corresponding $L-T$ Toeplitz matrix of order $T$ is given by

$$
A=\alpha\left(L_{T}\right)=\alpha(z)=\alpha_{0} I_{T}+\alpha_{1} L_{T}+\cdots+\alpha_{p-1} L_{T}^{p-1}+\alpha_{p} L_{T}^{p} .
$$

An example of this matrix is as follows:

$$
A=\left[\begin{array}{ccccccccc}
\alpha_{0} & 0 & \cdots & 0 & 0 & 0 & \cdots & 0 & 0 \\
\alpha_{1} & \alpha_{0} & \cdots & 0 & 0 & 0 & \cdots & 0 & 0 \\
\vdots & \vdots & \ddots & \vdots & \vdots & \vdots & \ddots & \vdots & \\
\alpha_{p-1} & \alpha_{p-2} & \cdots & \alpha_{0} & 0 & 0 & \cdots & 0 & 0 \\
\hline \alpha_{p} & \alpha_{p-1} & \cdots & \alpha_{1} & \alpha_{0} & 0 & \cdots & 0 & 0 \\
0 & \alpha_{p} & \cdots & \alpha_{2} & \alpha_{1} & \alpha_{0} & \cdots & 0 & 0 \\
\vdots & \vdots & \ddots & \vdots & \vdots & \vdots & \ddots & \vdots & \vdots \\
0 & 0 & \cdots & \alpha_{p} & \alpha_{p-1} & \alpha_{p-2} & \cdots & \alpha_{0} & 0 \\
0 & 0 & \cdots & 0 & \alpha_{p} & \alpha_{p-1} & \cdots & \alpha_{1} & \alpha_{0}
\end{array}\right]=\left[\begin{array}{c}
Q_{*}^{\prime} \\
Q^{\prime}
\end{array}\right]
$$

Here, the matrix has been partitioned with the first $p$ rows in $Q_{*}^{\prime}$ and with the remaining $T-p$ rows in $Q^{\prime}$. The latter contains the complete set of polynomial coefficients within each of its rows. (Notwithstanding a feature of this example, it should not be supposed that, in general, $Q^{\prime}$ comprises $p+1$ rows.)

By removing the first (or the last) $q$ rows and columns from $Q^{\prime}$, we obtain a submatrix that has the same essential structure as $Q^{\prime}$, with the coefficients of $\alpha(z)$ in each row and with $\alpha_{p}$ as its leading element. On removing from $Q^{\prime}$ the $q$ leading rows alone, we obtain a matrix with an additional $q$ leading columns that contain zero-valued elements.

A typical use of the structure of (4) concerns the application of $p$-fold differencing operator to a vector $y$ of order $T$ generated by an ARIMA process with $p$ roots of unity. The purpose is to reduce the vector to one that would have been generated by a stationary process.

The matrix version of the $p$-fold difference operator is $\nabla^{p}=\left(I_{T}-L_{T}\right)^{p}$. This matrix may be partitioned as $\nabla^{p}=\left[Q_{*}, Q\right]^{\prime}$ where $Q_{*}^{\prime}=\left[I_{p}, 0\right] \nabla^{p}$ has $p$ rows and where $Q^{\prime}=\left[0, I_{T-p}\right] \nabla^{p}$ has $T-p$ rows. The inverse may be partitioned conformably such that $\nabla^{-p}=\left[S_{*}, S\right]$, where $S_{*}$ and $S$ have $p$ and $T-p$ columns, respectively.

When the differencing operator is applied to a vector $y$, the first $p$ elements of the product, which are in $d_{*}$, are not true differences and they are liable to be discarded:

$$
\nabla^{p} y=\left[\begin{array}{l}
Q_{*}^{\prime} \\
Q^{\prime}
\end{array}\right] y=\left[\begin{array}{c}
d_{*} \\
d
\end{array}\right]
$$

However, if the elements of $d_{*}$ are available, then the vector $y$ can be recovered via the equation

$$
y=S_{*} d_{*}+S d .
$$

The columns of the matrix $S_{*}$ provide a basis for the set of polynomials of degree $p-1$ defined over the integer values $t=0,1, \ldots, T-1$. Therefore, $f=S_{*} d_{*}$ 


\section{D.S.G. POLLOCK: Econometric Signal Extraction}

is a vector of polynomial ordinates, whilst $d_{*}$ can be regarded as a vector of $p$ polynomial parameters.

The trend that underlies the data can be estimated via the polynomial ordinates that are obtained projecting the data vector $y$ onto the manifold of $S_{*}$ to give

$$
f=S_{*}\left(S_{*}^{\prime} S_{*}\right)^{-1} S_{*}^{\prime} y .
$$

Given that $Z=\left[Q, S_{*}\right]$ is a square matrix of full rank with $Q^{\prime} S_{*}=0$, it follows that

$$
Z\left(Z^{\prime} Z\right)^{-1} Z^{\prime}=Q\left(Q^{\prime} Q\right)^{-1} Q^{\prime}+S_{*}\left(S_{*}^{\prime} S_{*}\right)^{-1} S_{*}^{\prime}=I .
$$

Therefore

$$
\begin{aligned}
f & =y-Q\left(Q^{\prime} Q\right)^{-1} Q^{\prime} y \\
& =y-u,
\end{aligned}
$$

where $u=Q\left(Q^{\prime} Q\right)^{-1} Q^{\prime} y$ is the vector of regression residuals.

\section{A Model of Unobserved Components}

A typical econometric time series is a trend that is affected by seasonal fluctuations and irregular disturbances. A vector of $T$ observations is described by the equation

$$
y=\xi+\eta=(\rho+\kappa)+\eta,
$$

where $\rho$ is the trend component, $\kappa$ is the seasonal component and $\eta$ is the irregular component. The latter is typically regarded as having been generated by a whitenoise process, of which the expected value $E(\eta)$ and the variance-covariance or dispersion matrix $D(\eta)$ are

$$
E(\eta)=0 \quad \text { and } \quad D(\eta)=\Omega_{\eta}=\sigma_{\eta}^{2} I_{T} .
$$

The composite vector $\xi=\rho+\kappa$ is described as the nonstationary component.

The estimates of the components $\xi, \rho, \kappa$ and $\eta$ may be denoted by the corresponding Roman letters $x, r, k$ and $h$; so that the estimated version of the equation (10) would be

$$
y=x+h=(r+k)+h .
$$

The composite operator $\Delta=\nabla^{2} \Sigma$ that is typically employed in reducing $y$ to stationarity is the product of the detrending operator $\nabla^{p}=\left(I_{T}-L_{T}\right)^{p}$, with $p=2$, and the deseaonalising operator $\Sigma=\left(I-L_{T}^{s}\right)\left(I-L_{T}\right)^{-1}=I+L_{T}+\cdots+L_{T}^{s-1}$, where $s$ is, typically, the number of months or quarters in a year. The operator is partitioned as $\Delta=\left[Q_{*}, Q\right]^{\prime}$, where $Q_{*}^{\prime}$ has $p+s-1$ rows and $T$ columns, and there is an inverse matrix $\Delta^{-1}=\left[S_{*}, S\right]$, where $S_{*}$ has $p+s-1$ columns and $T$ rows.

Applying the operator $\Delta$ to equation (10) gives

$$
g=Q^{\prime} y=Q^{\prime}(\rho+\kappa)+Q^{\prime} \eta \quad \text { and } \quad g_{*}=Q_{*}^{\prime} y .
$$

The components $Q^{\prime} \rho$ and $Q^{\prime} \kappa$ are over-differenced, and $\rho$ and $\kappa$ may be reduced to stationarity instead by the component operators $\nabla^{2}$ and $\Sigma$ respectively. Thus

$$
\nabla^{2} \rho=\left[\begin{array}{c}
Q_{\rho *}^{\prime} \\
Q_{\rho}^{\prime}
\end{array}\right] \rho=\left[\begin{array}{c}
\varepsilon_{*} \\
\varepsilon
\end{array}\right] \quad \text { and } \Sigma \kappa=\left[\begin{array}{c}
Q_{\kappa *}^{\prime} \\
Q_{\kappa}^{\prime}
\end{array}\right] \kappa=\left[\begin{array}{c}
\zeta_{*} \\
\zeta
\end{array}\right] .
$$




\section{D.S.G. POLLOCK: Econometric Signal Extraction}

The resulting sub vectors $\varepsilon$ and $\zeta$ are characterised by stationary distributions with

$$
E(\varepsilon)=\mu, \quad D(\varepsilon)=\Omega_{\varepsilon} \quad \text { and } \quad E(\zeta)=0, \quad D(\zeta)=\Omega_{\zeta},
$$

and it is assumed that the elements of $\varepsilon$ and $\zeta$ are statistically independent of the initial conditions within $\varepsilon_{*}$ and $\zeta_{*}$.

We define $\nabla^{-2}=\left[S_{\rho *}, S_{\rho}\right]$ and $\Sigma^{-1}=\left[S_{\kappa *}, S_{\kappa}\right]$. Given the appropriate initial conditions, it is possible to recover the nonstationary vector $\xi$ and its component vectors via

$$
\begin{aligned}
\xi & =\rho+\kappa \\
& =\left[\begin{array}{ll}
S_{\rho *} & S_{\rho}
\end{array}\right]\left[\begin{array}{c}
\varepsilon_{*} \\
\varepsilon
\end{array}\right]+\left[\begin{array}{ll}
S_{\kappa *} & S_{\kappa}
\end{array}\right]\left[\begin{array}{c}
\zeta_{*} \\
\zeta
\end{array}\right] .
\end{aligned}
$$

The parameter $\mu=E(\varepsilon)$ of (15) may be described as the drift parameter of the stochastic trend component $\rho$. This should be present in case the trend is modelled by a first-order random walk, which implies that $p=1$. In that case, $f=S_{\rho *} \varepsilon_{*}$ is a constant function. When $p>1, f$ contains the ordinates of a polynomial of degree $p-1$, which automatically imparts a drift to $\rho$. Since we may assume that $p>1$, we may set $\mu=0$.

The object is to estimate the components $\eta, \rho$ and $\kappa$ of equation (10) by taking account of the distributional properties of the stationary vectors $\eta, \varepsilon$ and $\zeta$ and by using the information that is contained within $g=Q^{\prime} y$ of (13).

The strategy is to decompose $y=x+h$ into $x$ and $h$, which are the estimates of the nonstationary component $\xi$ and of the irregular component $\eta$, respectively. Thereafter, $x=r+k$ is decomposed into the estimate $r$ of the trend component $\rho$ and the estimate $k$ of the seasonal component $\kappa$. The latter estimates are derived from the estimates $e$ and $z$ of the vectors $\varepsilon$ and $\zeta$, which contain the elements of the relevant forcing functions.

There are

$$
Q^{\prime}=\left[\begin{array}{ll}
0 & I_{n}
\end{array}\right] \Delta \text { and } \rho=\nabla^{-2}\left[\begin{array}{c}
\varepsilon_{*} \\
\varepsilon
\end{array}\right],
$$

where $n=T-p-s+1$. Therefore, since $\Delta=\Sigma \nabla^{2}$ implies that $\Delta \nabla^{-2}=\Sigma$, the first component of $g$ is

$$
Q^{\prime} \rho=\left[\begin{array}{ll}
0 & I_{n}
\end{array}\right] \Delta \nabla^{-2}\left[\begin{array}{c}
\varepsilon_{*} \\
\varepsilon
\end{array}\right]=\left[\begin{array}{ll}
0 & I_{n}
\end{array}\right] \Sigma\left[\begin{array}{c}
\varepsilon_{*} \\
\varepsilon
\end{array}\right]=Q_{\kappa_{\Delta}}^{\prime} \varepsilon,
$$

Here, $\left[0, I_{n}\right] \Sigma$ is a matrix of order $(T-p-s+1) \times T$, which is obtained by deleting $p+s-1$ rows from $\Sigma$. It has more than $p$ leading columns of zero-valued elements, which is the number of elements in $\varepsilon_{*}$. Deleting $p$ columns of zeros from this matrix produces the matrix $Q_{\kappa_{\triangle}}^{\prime}$.

An analogous expression for the second component of $g$ is

$$
Q^{\prime} \kappa=\left[\begin{array}{ll}
0 & I_{n}
\end{array}\right] \Delta \Sigma^{-1}\left[\begin{array}{c}
\zeta_{*} \\
\zeta
\end{array}\right]=\left[\begin{array}{ll}
0 & I_{n}
\end{array}\right] \nabla^{2}\left[\begin{array}{c}
\zeta_{*} \\
\zeta
\end{array}\right]=Q_{\rho_{\Delta}}^{\prime} \zeta .
$$

According to the Wiener-Kolmogorov principle, the estimates of the components of $y$ are provided by their conditional expectations. The estimate of the irregular component $\eta$ is given by

$$
h=E(\eta \mid g)=C(\eta, g) D^{-1}(g) g,
$$


wherein there are

$$
C(\eta, g)=\Omega_{\eta} Q \quad \text { and } \quad D(g)=\Omega_{\eta}+Q_{\kappa_{\triangle}}^{\prime} \Omega_{\varepsilon} Q_{\kappa_{\triangle}}^{\prime}+Q_{\rho_{\triangle}}^{\prime} \Omega_{\zeta} Q_{\rho_{\triangle}}^{\prime}
$$

Then, an estimate of $\xi=\rho+\kappa=y-\eta$ is given by

$$
x=E(\xi \mid g)=y-h,
$$

from which are derived

$$
d_{*}=Q_{*}^{\prime} x \quad \text { and } \quad d=Q^{\prime} x .
$$

Having extracted the irregular component from the data, it is now a matter of dividing the residue between the two nonstationary components-i.e. the trend $\rho$ and the seasonal component $\kappa$. For this purpose, the following estimates of the two forcing functions $\varepsilon$ and $\zeta$ are required:

$$
e=E(\varepsilon \mid g)=C(\varepsilon, g) D^{-1}(g) g \quad \text { and } \quad z=E(\zeta \mid g)=C(\zeta, g) D^{-1}(g) g .
$$

Here,

$$
C(\varepsilon, g)=\Omega_{\varepsilon} Q_{\kappa_{\triangle}} \quad \text { and } \quad C(\zeta, g)=\Omega_{\zeta} Q_{\rho \triangle}
$$

By replacing the unknown elements of (16) by their various estimates, we get

$$
\begin{aligned}
x & =r+k \\
& =\left[\begin{array}{ll}
S_{\rho *} & S_{\rho}
\end{array}\right]\left[\begin{array}{c}
e_{*} \\
e
\end{array}\right]+\left[\begin{array}{ll}
S_{\kappa *} & S_{\kappa}
\end{array}\right]\left[\begin{array}{c}
z_{*} \\
z
\end{array}\right] .
\end{aligned}
$$

Rearranging this and using $x=S_{*} d_{*}+S d$ gives

$$
x=\left[\begin{array}{ll}
S_{*} & S
\end{array}\right]\left[\begin{array}{c}
d_{*} \\
d
\end{array}\right]=\left[\begin{array}{ll}
S_{\rho *} & S_{\kappa *}
\end{array}\right]\left[\begin{array}{l}
e_{*} \\
z_{*}
\end{array}\right]+\left[\begin{array}{ll}
S_{\rho} & S_{\kappa}
\end{array}\right]\left[\begin{array}{l}
e \\
z
\end{array}\right],
$$

from which

$$
S_{*} d_{*}=\left[\begin{array}{ll}
S_{\rho *} & S_{\kappa *}
\end{array}\right]\left[\begin{array}{c}
e_{*} \\
z_{*}
\end{array}\right] \quad \text { and } \quad S d=\left[\begin{array}{ll}
S_{\rho} & S_{\kappa}
\end{array}\right]\left[\begin{array}{l}
e \\
z
\end{array}\right] .
$$

The first of these equations can be solved for $e_{*}$ and $z_{*}$, for which purpose only the first $p+q-1$ rows of the system are required. Thereafter, the estimates for $\rho$ and $\kappa$ are given by

$$
r=S_{\rho *} e_{*}+S_{\rho} e \quad \text { and } \quad k=S_{\kappa *} z_{*}+S_{\kappa} z
$$

What has been recounted here is essentially the method proposed by Bell (1984) and elaborated in an unpublished paper of Bell and Hillmer (1988), which has been referenced recently by McElroy and Sutcliffe (2006).

\section{Linear Stochastic Models}

There are three alternative, but closely related, linear stochastic models that can be accommodated within the equations for signal extraction that have been provided in the foregoing section. 


\section{D.S.G. POLLOCK: Econometric Signal Extraction}

To express these models in the most direct manner, it is appropriate to adopt the $z$-transform algebra that pertains to doubly-infinite sequences. However, it is commonly supposed that the fundamental forcing functions of the models take the form of stationary white-noise sequences. When the unit root autoregressive operators of the models are applied to such sequences, the results will be unbounded, almost surely.

To avoid the mathematical difficulty of unbounded sequences, it might be convenient to assume that, whereas they are defined on doubly-infinite index sets, the forcing functions take nonzero values only on a finite set of contiguous indices. This is, of course, in contradiction to the common statistical assumptions. However, in this way, the $z$-transform algebra becomes acceptable.

\section{The Airline Passenger Model and TRAMO-SEATS}

The first of the linear stochastic models to be considered is the airline passenger model of Box and Jenkins (1976). This is represented by the equation

$$
y(z)=\frac{N(z)}{\Delta(z)} \varepsilon(z)=\left\{\frac{(1-\phi z)\left(1-\theta^{s} z^{s}\right)}{(1-z)\left(1-z^{s}\right)}\right\} \varepsilon(z),
$$

where $N(z)$ and $\Delta(z)$ are polynomial operators and $y(z)$ and $\varepsilon(z)$ are, respectively, the $z$-transforms of the output sequence $y(t)=\left\{y_{t} ; t=0, \pm 1, \pm 2, \ldots\right\}$ and of the input sequence $\varepsilon(t)=\left\{\varepsilon_{t} ; t=0, \pm 1, \pm 2, \ldots\right\}$ of unobservable white-noise disturbances. The integer $s$ stands for the number of periods in the year, which are $s=4$ for quarterly data and $s=12$ for monthly data. In deriving the filters, an arbitrary value can be attributed to the variance of the input sequence; and it is convenient to set this to unity.

Given the identity $1-z^{s}=(1-z) \Sigma(z)$, where $\Sigma(z)=1+z+\cdots+z^{s-1}$ is the seasonal summation operator, it follows that the denominator polynomial, which is the $z$-transform version of the composite difference operator of the previous section, may be expressed as

$$
\Delta(z)=(1-z)\left(1-z^{s}\right)=\nabla^{2}(z) \Sigma(z)
$$

where $\nabla(z)=1-z$ is the backward difference operator. The polynomial $\Sigma(z)$ has zeros at the points $\exp \{\mathrm{i}(2 \pi / s) j\} ; j=1,2, \ldots, s-1$, which are located on the circumference of the unit circle in the complex plane at angles from the horizontal that correspond to the fundamental seasonal frequency $\omega_{s}=2 \pi / s$ and its harmonics.

The TRAMO-SEATS program effects a decomposition of the data into a seasonal component and a non-seasonal component that are described by statistically independent processes driven by separate white-noise forcing functions. It espouses the principal of canonical decompositions that has been expounded by Hillmer and Tiao (1982).

The first step in this decomposition entails the following partial-fraction decomposition of the generating function of the autocovariances of $y(t)$ :

$$
\frac{N\left(z^{-1}\right) N(z)}{\Delta\left(z^{-1}\right) \Delta(z)}=\frac{U^{*}\left(z^{-1}\right) U^{*}(z)}{\nabla^{2}\left(z^{-1}\right) \nabla^{2}(z)}+\frac{V^{*}\left(z^{-1}\right) V^{*}(z)}{\Sigma\left(z^{-1}\right) \Sigma(z)}+\phi \theta^{s} .
$$




\section{D.S.G. POLLOCK: Econometric Signal Extraction}

The decomposition is effected most readily by casting the LHS of the equation above in the form of a trigonometrical polynomial. Here, $\phi \theta^{s}$ is the quotient of the division of $N\left(z^{-1}\right) N(z)$ by $\Delta\left(z^{-1}\right) \Delta(z)$, which must occur before the remainder, which will be a proper fraction, can be decomposed.

In the preliminary decomposition of (32), the first term on the RHS corresponds to the trend component, the second term corresponds to the seasonal component and the third term corresponds to the irregular component. Hillmer and Tiao have provided expressions for the numerators of the RHS, which are somewhat complicated, albeit that the numerators can also be found by numerical means.

When $z=e^{\mathrm{i} \omega}$, equation (32) provides the spectral ordinates of the process and of its components at the frequency value of $\omega$. The corresponding spectral density functions are obtained by letting $\omega$ run from 0 to $\pi$. The quotient $\phi \theta^{s}$ corresponds to the spectrum a white-noise process, which is constant over the frequency range.

The principal of canonical decomposition proposes that the estimates of the trend and of the seasonal component should be devoid of any elements of white noise. Therefore, their spectra must be zero-valued at some point in the interval $[0, \pi]$. Let $\lambda_{\rho}$ and $\lambda_{\kappa}$ be the minima of the spectral density functions associated with the trend and the seasonal components respectively. By subtracting these values from their respective components, a revised decomposition is obtained that fulfils the canonical principal. This is

$$
\frac{N\left(z^{-1}\right) N(z)}{\Delta\left(z^{-1}\right) \Delta(z)}=\frac{U\left(z^{-1}\right) U(z)}{\nabla^{2}\left(z^{-1}\right) \nabla^{2}(z)}+\frac{V\left(z^{-1}\right) V(z)}{\Sigma\left(z^{-1}\right) \Sigma(z)}+\lambda,
$$

where $\lambda=\phi \theta^{s}+\lambda_{\rho}+\lambda_{\kappa}$.

The Wiener-Kolmogorov principle of signal extraction indicates that the filter that serves to extract the trend from the data sequence $y(t)$ should take the form of

$$
\begin{aligned}
\beta_{T}(z) & =\frac{U\left(z^{-1}\right) U(z)}{\nabla^{2}\left(z^{-1}\right) \nabla^{2}(z)} \times \frac{\Delta\left(z^{-1}\right) \Delta(z)}{N\left(z^{-1}\right) N(z)} \\
& =\frac{U\left(z^{-1}\right) U(z)}{N\left(z^{-1}\right) N(z)} \times \Sigma\left(z^{-1}\right) \Sigma(z) .
\end{aligned}
$$

This is the ratio of the autocovariance generating function of the trend component to that of the process as a whole. This filter nullifies the seasonal component in the process of extracting a trend that is relatively free of high-frequency elements. The nullification of the seasonal component is due to the factor $\Sigma(z)$.

The gain of the trend-extraction filter is depicted in Figure 1. Here, the values of $\phi=0.4$ and $\theta^{12}=0.6$ that determine the polynomial $N(z)$ are the estimates of Box and Jenkins (1976). The filter is an appropriate device for seasonal adjustment if the high-frequency elements that it serves to attenuate are liable to be regarded as a noisy contamination of no economic significance. In that case, as we shall propose later, it might be best to be remove them completely from the data.

The seasonal-adjustment filter, which nullifies the seasonal component without further attenuating the high-frequency elements of the data, is marginally more complicated. Define

$$
\frac{W\left(z^{-1}\right) W(z)}{\nabla^{2}\left(z^{-1}\right) \nabla^{2}(z)}=\frac{U\left(z^{-1}\right) U(z)}{\nabla^{2}\left(z^{-1}\right) \nabla^{2}(z)}+\lambda
$$




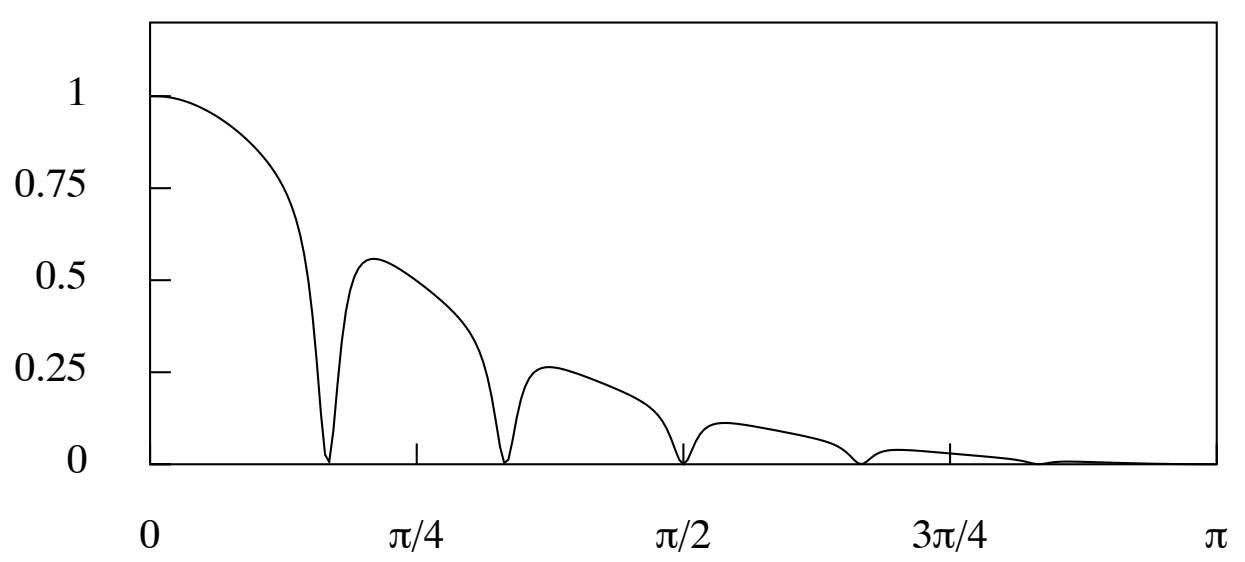

Figure 1. The gain of the trend extraction filter associated with the monthly airline passenger model.

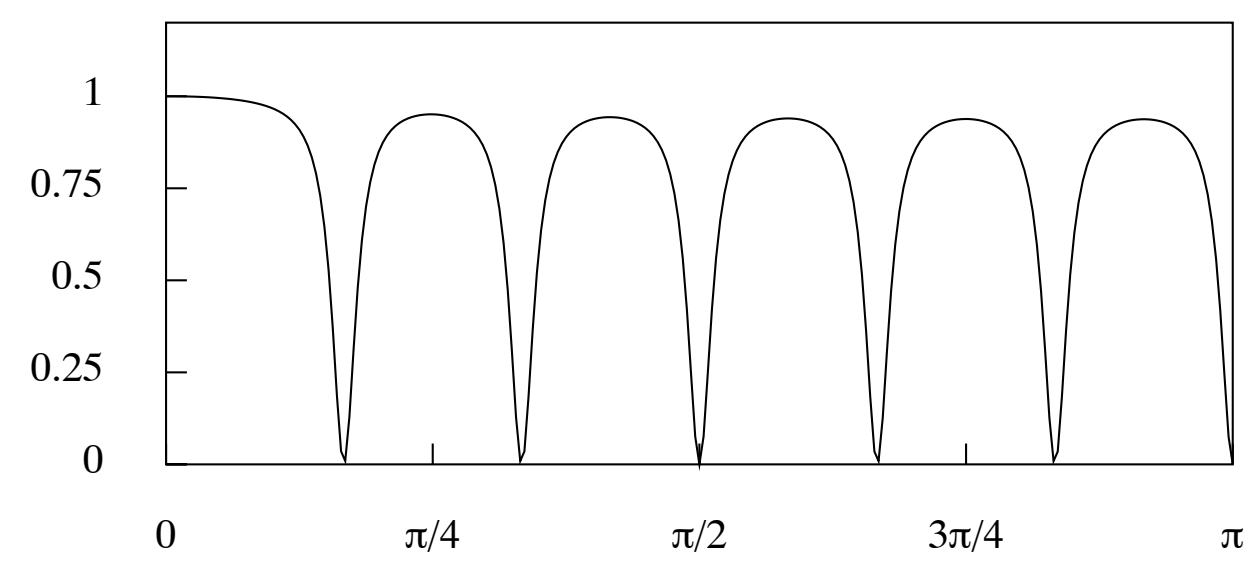

Figure 2. The gain of the seasonal-adjustment filter associated with the monthly airline passenger model.

Then, the seasonal-adjustment filter is

$$
\beta_{A}(z)=\frac{W\left(z^{-1}\right) W(z)}{N\left(z^{-1}\right) N(z)} \times \Sigma\left(z^{-1}\right) \Sigma(z) .
$$

The gain of this filter is shown in Figure 2. Further examples of its gain, for various values of the parameters $\phi$ and $\theta$, are provided in a paper of Findlay (2005).

The filters derived from the canonical decomposition of the airline passenger model can be derived from a meta-model defined by the equation

$$
\begin{aligned}
y(z) & =\frac{U(z)}{\nabla^{2}(z)} \varepsilon(z)+\frac{V(z)}{\Sigma(z)} \zeta(z)+\eta(z) \\
& =\rho(z)+\kappa(z)+\eta(z) .
\end{aligned}
$$

Within this model, unit variances would be attributed to the stochastic sequences $\varepsilon(t)$ and $\zeta(t)$, whereas $\lambda$ would be the variance of $\eta(t)$.

Whereas no explicit expressions are available for $U(z)$ and $V(z)$, the expressions for $\Omega_{\varepsilon}(z)=U\left(z^{-1}\right) U(z)$ and $\Omega_{\zeta}(z)=V\left(z^{-1}\right) V(z)$ have been provided by 
Hillmer and Tao (1982). On substituting the matrices $L_{T}$ and $L_{T}^{\prime}$ for $z$ and $z^{-1}$, respectively, within these expressions, the corresponding finite-sample dispersion matrices of equation (15) are derived. Thus, the necessary elements for the finitesample implementation of the filters according to the methods described in section 4 are readily available.

\section{The Structural Times Series Model and STAMP}

The so-called structural time series model, which is the basis of the STAMP program, can be expressed via the equation

$$
\begin{aligned}
y(z) & =\left\{\frac{z \nu(z)}{\nabla^{2}(z)}+\frac{\xi(z)}{\nabla(z)}\right\}+\frac{\zeta(z)}{\Sigma(z)}+\eta(z) \\
& =\rho(z)+\kappa(z)+\eta(z),
\end{aligned}
$$

wherein $\zeta(z), \xi(z), \nu(z)$ and $\eta(z)$ are the $z$-transforms of independently distributed white-noise sequences. The structural model is well identified and its estimation requires only the determination of the variances of the four white-noise processes.

The first two terms on the RHS of (38) can be combined to give

$$
\rho(z)=\frac{z \nu(z)}{\nabla^{2}(z)}+\frac{\xi(z)}{\nabla(z)}=\frac{(1-\mu z)}{\nabla^{2}(z)} \varepsilon(z),
$$

which stands for a doubly integrated first-order moving-average process wherein $\varepsilon(t)$ is also a white-noise process. This component of the model is intended to capture the trend in the data.

By combining the terms of equation (38), an ARIMA model is derived that has the same autoregressive operator as the airline passenger model as well as a moving average operator of the same order. Therefore, notwithstanding the different restrictions that are applied to the moving-average coefficients, it is unsurprising that these models should deliver similar estimates of the autocovariance function, as has been observed by Maravall (1985).

The differences between the filters derived from the STAMP model and those that are derived from the airline passenger model are mainly on account of the principle of canonical decomposition that is applied to the latter. However, there is no reason why the principle cannot be applied to the STAMP model as well.

Figure 3 shows, via the continuous line, the gain of the trend-estimation filter that is derived from the STAMP model. Also shown on diagram, via the broken line, is the canonical version of the filter. This is obtained by subtracting the white-noise contaminations from the trend and the seasonal components and by assigning them to the irregular component.

It will be observed that frequency responses of the canonical filters of the airline passenger model and of the STAMP model are similar, albeit that the response of the STAMP filter has narrower notches at the seasonal frequency and its harmonics.

In practice, in the absence of the canonical modification, it will be found that, whenever the data sequence has a significant proportion of high-frequency variation, the trend that is estimated by the STAMP procedure will have a rougher profile than the estimate of TRAMO-SEATS. (See Pollock, 2002) 


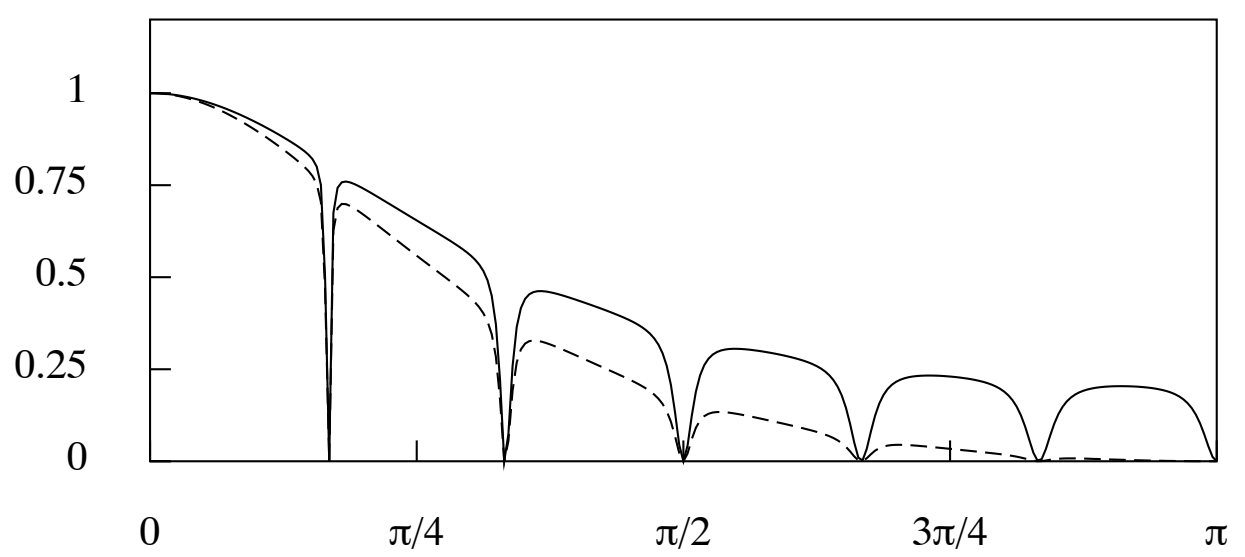

Figure 3. The gain of the trend extraction filter associated with the structural time series model (the solid line) together with that of the canonical version of the filter (the broken line).

\section{The Polynomial Trend and IDEOLOG}

A further method that is aimed at the seasonal adjustment of the data presumes that the underlying trend can be represented adequately by a polynomial function. The equation of the model is

$$
\begin{aligned}
y(z) & =\rho(z)+\frac{\Sigma(\theta z)}{\Sigma(z)} \zeta(z)+\eta(z) \\
& =\rho(z)+\kappa(z)+\eta(z),
\end{aligned}
$$

where $y(z)$ is the $z$-transform of the logarithms of the data and where $\rho(z)$ is the $z$-transform of a sequence of the ordinates of a polynomial that is fitted to the data. Also, $\zeta(z)$ and $\eta(z)$ correspond to mutually independent white-noise processes.

The model incorporates a representation of the seasonal component, which embodies the numerator polynomial

$$
\Sigma(\theta z)=1+\theta z+\theta^{2} z^{2}+\cdots+\theta^{s-1} z^{s-1}=\frac{\left(1-\theta^{s} z^{s}\right)}{(1-\theta z)}
$$

where $\theta<1$ is a positive number close to unity. Thus, in comparing equation (40) with equation (30) of the airline passenger model, we see that

$$
\frac{N(z)}{\Delta(z)}=\frac{(1-\phi z)(1-\theta z) \Sigma(\theta z)}{(1-z)^{2} \Sigma(z)}
$$

The additional factor $\{(1-\phi z)(1-\theta z)\} /(1-z)^{2}$ of the airline passenger model is the means by which it captures the trend in the data.

The seasonal-adjustment filter that extracts $\eta(z)$ from $g(z)=y(z)-\rho(z)$ is also the filter the extracts $\Sigma(z) \eta(z)$ from

$$
\Sigma(z) g(z)=\Sigma(\theta z) \zeta(z)+\Sigma(z) \eta(z)
$$




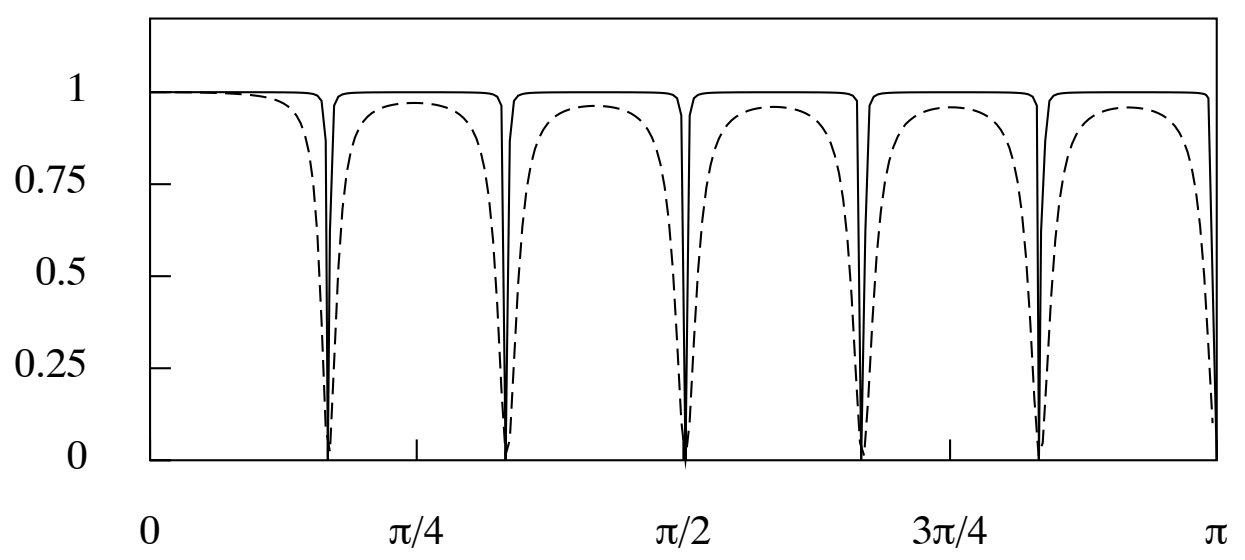

Figure 4. The gain of the seasonal adjustment filter associated with the monthly version of the model with a polynomial trend. The solid line corresponds to the parameters $\theta=0.6$ and $\lambda=\sigma_{\eta}^{2} / \sigma_{\zeta}^{2}=0.125$ and the broken line corresponds to the parameters $\theta=0.6$ and $\lambda=0.125$.

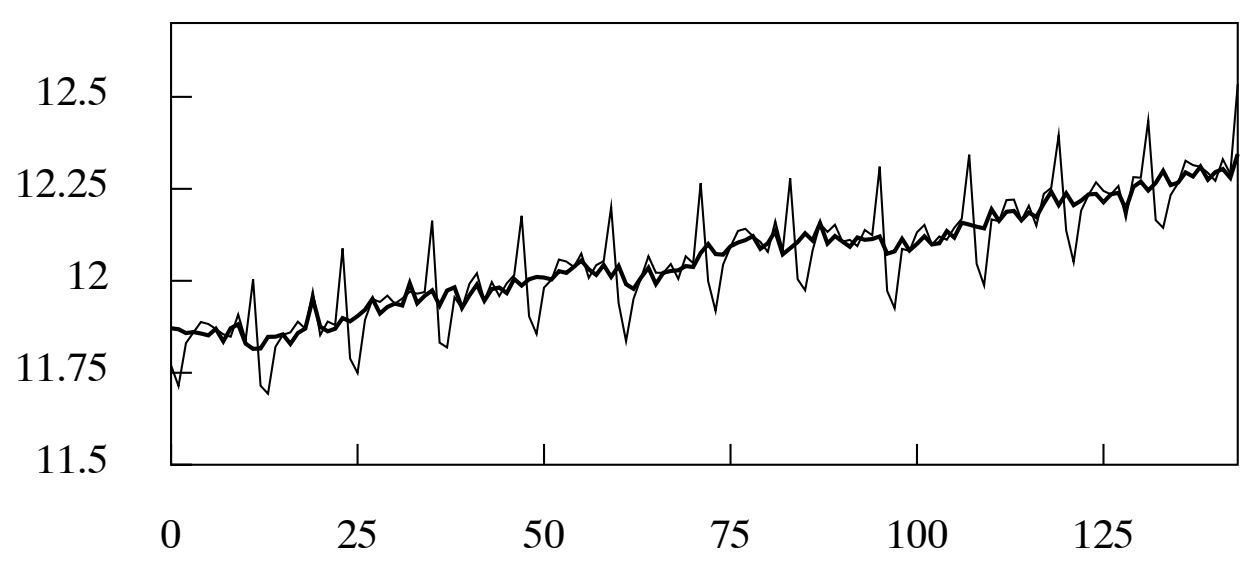

Figure 5. The effect of applying seasonal adjustment filter of the model with a polynomial trend to the logarithms of the monthly index of U.S. total sales from January 1953 to December 1964. The parameters of the model are $\theta=0.99$ and $\lambda=\sigma_{\eta}^{2} / \sigma_{\zeta}^{2}=0.125$.

The $z$-transform of the filter, which is derived according to the Wiener-Kolmogorov principle, is

$$
\beta_{C}(z)=\frac{\sigma_{\eta}^{2} \Sigma(z) \Sigma\left(z^{-1}\right)}{\sigma_{\eta}^{2} \Sigma(z) \Sigma\left(z^{-1}\right)+\sigma_{\zeta}^{2} \Sigma(\theta z) \Sigma\left(\theta z^{-1}\right)} .
$$

Setting $z=\exp \{-\mathrm{i} \omega\}$ and letting $\omega$ run from 0 to $\pi$ generates the frequency response of the filter. The modulus or gain is plotted in Figure 4 for the case where $\theta=0.99$ and $\lambda=\sigma_{\eta}^{2} / \sigma_{\zeta}^{2}=0.125$, via the solid line, and for the case where $\lambda=0.6$, via the broken line. There is hardly a difference between the gain in Figure 4, in the latter case, and that of Figure 2, which relates to the seasonal-adjustment filter associated with the airline passenger model.

In fact, $\beta_{C}(z)$ is a classic comb filter in which the zeros of the polynomial $\Sigma(z)$, which are located on the perimeter of the unit circle, are balanced by poles of the polynomial $\Sigma(\theta z)$ that fall on the same radii but which are located within 


\section{D.S.G. POLLOCK: Econometric Signal Extraction}

the circle at a short distance from the perimeter.

The poles counteract the effects of the zeros, except in the neighbourhoods of the seasonal frequencies and its harmonics, where the zeros account for the notches in the gain function. As $\theta \rightarrow 1$, the gain of the filter approaches unity at all points other than those in the vicinities of the zeros, which eliminate the elements at the seasonal frequency and its harmonics.

To derive the finite-sample version of the Wiener-Kolmogorov filter, we may consider a data vector $g=\left[g_{0}, g_{1}, \ldots, g_{T-1},\right]^{\prime}$ that has a seasonal component $\kappa$ and a noise component $\eta$ :

$$
g=y-\rho=\kappa+\eta \text {. }
$$

To cast the equations into a form analogous to (43), it is necessary to define the matrix counterparts of the polynomial operators $\Sigma(\theta z)$ and $\Sigma(z)$. By replacing $z$ in the polynomial operators by the matrix $L_{T}=\left[e_{1}, e_{2}, \ldots, e_{T-1}, 0\right]$, we get

$$
\Sigma\left(\theta L_{T}\right)=\left[\begin{array}{c}
Q_{* \Theta}^{\prime} \\
Q_{\Theta}^{\prime}
\end{array}\right] \quad \text { and } \quad \Sigma\left(L_{T}\right)=\left[\begin{array}{c}
Q_{* \Sigma}^{\prime} \\
Q_{\Sigma}^{\prime}
\end{array}\right]
$$

These are banded lower-triangular Toeplitz matrices with units on the diagonals. Since the matrices $Q_{* \Theta}^{\prime}$ and $Q_{* \Sigma}^{\prime}$ suffer from end effects, they are liable to be discarded leaving $Q_{\Theta}^{\prime}$ and $Q_{\Sigma}^{\prime}$, which are of order $(T-s+1) \times T$.

With these matrices, the following equation is formed, which is the matrix analogue of (43):

$$
Q_{\Sigma}^{\prime} g=Q_{\Theta}^{\prime} \zeta+Q_{\Sigma}^{\prime} \eta
$$

Here, $\zeta$ is a vector of order $T$ of white noise elements from the sequence $\zeta(t)$. A demonstration by Pollock (2007) serves to show that the minimum-mean-squareerror estimate of the vector $\eta$ is given by

$$
h=Q_{\Sigma}\left(Q_{\Sigma}^{\prime} Q_{\Sigma}+\lambda^{-1} Q_{\Theta}^{\prime} Q_{\Theta}\right)^{-1} Q_{\Sigma}^{\prime} g
$$

The matrix of the transformation mapping from $g$ to $h$ is seen to be the analogue of the filter function $\beta_{C}(z)$ of (44).

A simple procedure for calculating the estimate of $h$ is to solve the following equations in succession:

$$
\left(Q_{\Sigma}^{\prime} Q_{\Sigma}+\lambda^{-1} Q_{\Theta}^{\prime} Q_{\Theta}\right) b=Q_{\Sigma}^{\prime} g \quad \text { and } \quad h=Q_{\Sigma} b
$$

Since $Q_{\Sigma}^{\prime} Q_{\Sigma}$ and $Q_{\Theta}^{\prime} Q_{\Theta}$ correspond to the narrow-band dispersion matrices of moving-average processes, the solution to the first equation of (49) may be found via a Cholesky factorisation that sets $Q_{\Sigma}^{\prime} Q_{\Sigma}+\lambda^{-1} Q_{\Theta}^{\prime} Q_{\Theta}=G G^{\prime}$, where $G$ is a lower-triangular matrix with a limited number of nonzero bands. The system $G G^{\prime} b=Q_{\Sigma}^{\prime} g$ may be cast in the form of $G p=Q_{\Sigma}^{\prime} g$ and solved for $p$. Then, $G^{\prime} b=p$ can be solved for $b$.

Once the residual sequence from the polynomial detending has been seasonally adjusted, it can be added back to the interpolated polynomial. Although the seasonally-adusted sequence is ostensibly an estimate of the white-noise component $\eta$, its properties may differ from those of white noise. Any departures of the trend 


\section{D.S.G. POLLOCK: Econometric Signal Extraction}

from the fitted polynomial function will be captured by this sequence, and they will be restored to the trend when it is added to the polynomial.

Figure 5 shows the logarithms of 144 monthly observations on retail sales in U.S. for the period from January 1953 to December 1964. The data have been taken from the monograph of Shiskin et al. (1967) that described the X-11 program for seasonal adjustment. The figure also displays, via a bold line, a seasonally adjusted version of the data. This has been obtained by applying a filter to the residuals from a linear detrending and, thereafter, by adding the filtered sequence to the line.

The parameters of the filter are $\theta=0.99$ and $\lambda=0.125$. Its frequency response is depicted by the solid line in Figure 4. The filter nullifies only the elements at the seasonal frequency and at its harmonic frequencies. Elements at other frequencies are virtually unaffected.

The perception that the seasonal component can be attributed wholly to these few elements is based on an inspection of the periodogram of Figure 6. However, it might be proposed that a further element that is at a frequency slightly in excess of frequency $2 \pi / 3$, where the fourth spectral spike is to be found, should also be included in the synthesis of the seasonal component.

The alternative method of seasonal adjustment that we propose in the next section requires the elements that constitute the seasonal component to be identified by inspecting the periodogram of the detrended data. Once these elements have been identified, the seasonal component can be synthesised and subtracted thereafter from the original data sequence to produce a seasonally adjusted version.

\section{The Discrete Fourier Transform}

In a Fourier analysis, an arbitrary function is resolved into a weighted combination of sine and cosine functions or, alternatively, of complex exponential functions. The domain of such periodic functions is either the perimeter of a circle, or else it is the entire real line.

A finite data sequence that is subject to a Fourier analysis must be regarded as a single cycle of a periodic function. The periodicity is achieved either by mapping the sequence onto the circumference of a circle, or else by extending it indefinitely by successive replications.

If a data sequence contains a significant trend, then there will be a sharp disjunction at the point on the circle where the beginning of the sequence joins its end. Alternatively, there will be successive disjunctions in the periodic extension of the sequence at points where the end of one replication of the data joins the start of the next replication. Then, the effect will be to create the appearance of the serrated edge of a saw blade.

The spectrum of a saw tooth function has a one-over-f profile in the form of a rectangular hyperbola that extends from a high point adjacent to the zero frequency to a low point at the limiting Nyquist frequency of $\pi$ radians per sample interval. Such a profile is liable to mask the spectral information that is of genuine interest. Therefore, for a spectral analysis to be successful, the trend must be eliminated from the data at first.

The data can be detrended by differencing. Alternatively, a trend function can be interpolated into the data and the residual deviations can be subjected to the 


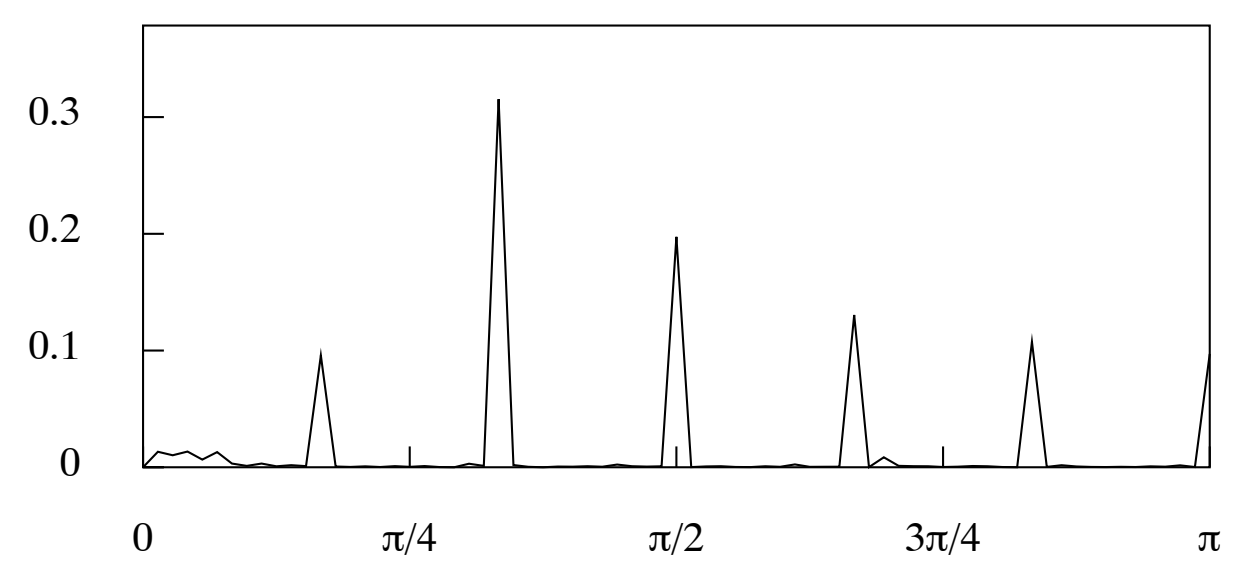

Figure 6. The periodogram of the residual sequence obtained from the linear detrending the logarithmic sales data.

analysis. In the context of the seasonal adjustment of the data, the latter approach is preferable, since the pattern of seasonal fluctuations can be obscured by taking differences. For the present, we shall assume that the detrended data are available in the vector $g$. Later, we shall describe various devices that can accompany the process of polynomial detrending, which are intended minimise the problem of the disjunctions.

It is more convenient to work with complex Fourier coefficients and with complex exponential functions in place sines and cosines. In these terms, the Fourier transform and its inverse are given by

$$
\gamma_{j}=\frac{1}{T} \sum_{t=0}^{T-1} g_{t} e^{-\mathrm{i} \omega_{j} t} \longleftrightarrow g_{t}=\sum_{j=0}^{T-1} \gamma_{j} e^{\mathrm{i} \omega_{j} t}
$$

where $\omega_{j}=2 \pi j / T$ is the $j$ th Fourier frequency, which, in the case of $j<T / 2$, relates to a sinusoidal element that completes $j$ cycles in the period spanned by the data. The conjugate frequencies $\omega_{T-j}$ are to be found within $\cos \left(\omega_{j}\right)=\left\{\exp \left(\omega_{j}\right)+\right.$ $\left.\exp \left(\omega_{T-j}\right)\right\} / 2$ and $\left.\cos \left(\omega_{j}\right)=\mathrm{i}\left\{\exp \left(\omega_{j}\right)-\exp \left(\omega_{T-j}\right)\right\} / 2\right)$, wherein $\exp \left(\omega_{T-j}\right)=$ $\exp \left(\omega_{-j}\right)$.

For a matrix representation of these transforms, one may define

$$
\begin{aligned}
& U=T^{-1 / 2}[\exp \{-\mathrm{i} 2 \pi t j / T\} ; t, j=0, \ldots, T-1], \\
& \bar{U}=T^{-1 / 2}[\exp \{\mathrm{i} 2 \pi t j / T\} ; t, j=0, \ldots, T-1],
\end{aligned}
$$

which are unitary complex matrices such that $U \bar{U}=\bar{U} U=I_{T}$. Then,

$$
\gamma=T^{-1 / 2} U g \quad \longleftrightarrow \quad g=T^{1 / 2} \bar{U} \gamma
$$

where $g=\left[g_{0}, g_{1}, \ldots g_{T-1}\right]^{\prime}$ and $\gamma=\left[\gamma_{0}, \gamma_{1}, \ldots \gamma_{T-1}\right]^{\prime}$ are the vectors of the data and of their spectral ordinates, respectively.

Figure 6 shows the periodogram of the residual sequence from a least-squares fitting of linear function to the logarithmic sales data. Although the trend has 


\section{D.S.G. POLLOCK: Econometric Signal Extraction}

been eliminated from the data, there remains a low-frequency component that is bounded by the fundamental seasonal frequency of $\pi / 6$.

There is liable to be a minor disjunction at the point in the circular wrapping of the data sequence where its starting point is adjacent to its end point or, equivalently, in the periodic extension of the data where one segment is followed by the next. The effects of this will be also felt throughout the residual sequence.

In the next section, it will be shown how the low-frequency component can be extracted from the residual sequence and how, in the process, the problem of the disjunction can be overcome. By adding the low-frequency component to the linear trend, a representation of the so-called trend-cyle component can be derived.

The method of frequency-domain filtering can also be applied to the problem of extracting the seasonal component. By subtracting this from the original logarithmic data sequence, a seasonally adjusted data sequence can be derived.

\section{Filtering in the Frequency Domain}

In the method of frequency-domain filtering, the ordinates of the Fourier transform of a stationary data sequence are modified or weighted according to the desired frequency response of the filter. Then, they are carried back to the time domain by an application of the inverse Fourier transform to form the filtered sequence.

Let $J$ be a diagonal weighting matrix of order $T$. In the case of an ideal frequency selective filter, the diagonal elements of $J$ consists of units that correspond to the frequencies of the pass band and zeros that correspond to the frequencies of the stop band. Then, the selected Fourier ordinates are the nonzero elements of the vector $J \gamma$ and the filtered sequence is

$$
w=\bar{U} J U g=\Psi g .
$$

Here, $\bar{U} J U=\Psi=\left[\psi_{|i-j|}^{\circ} ; i, j=0, \ldots, T-1\right]$ is a circulant matrix of the filter coefficients that would result from wrapping the infinite sequence of the coefficients of the ideal bandpass filter around a circle of circumference $T$ and adding the overlying elements. Thus

$$
\psi_{k}^{\circ}=\sum_{q=-\infty}^{\infty} \psi_{q T+k} .
$$

Applying the wrapped filter to the finite data sequence via a circular convolution is equivalent to applying the original filter to an infinite periodic extension of the data sequence. In practice, the wrapped coefficients of the time-domain filter matrix $\Psi$ would be obtained from the Fourier transform of the vector of the diagonal elements of the matrix $J$. However, it is more efficient to perform the filtering by operating upon the Fourier ordinates in the frequency domain, which is how the program IDEOLOG operates.

\section{Tapering and Extrapolations}

Various devices are available within IDEOLOG for ensuring that, when the data sequence is wrapped around the circumference of a circle, there is no disjunction at the point where its head joints its tail. 


\section{D.S.G. POLLOCK: Econometric Signal Extraction}

The conventional means of avoiding such disjunctions is to taper a detrended and mean-adjusted sequence so that both ends decay to zero. (See Bloomfield 1976, for example.) The disadvantage of this recourse is that it falsifies the data at the ends of the sequence, which is particularly inconvenient if, as is often the case in economics, attention is focussed on the most recent data. To avoid this difficulty, the tapering can be applied to some extrapolations, which can be added to the data, after it has been subject to a preliminary detrending.

The preliminary detrending can be achieved by interpolating a polynomial function of time of by using the Leser or Hodrick-Prescott filter. (This filter, which is commonly attributed by economists to Hodrick and Prescott $(1980,1997)$, was expounded by Leser (1961) in an earlier publication.) The interpolated function should be a stiff one containing only periodic elements of the lowest frequencies. It is also desirable that the function should pass through the midst of the scatters of points at either end of the data sequence. For this purpose, a method of weighted least-squares polynomial regression can be used that allows extra weight to be placed upon the initial and the final runs of observations.

The method of weighted least-squares can also be used in the context of the Leser filter. Here, an additional flexibility is available by allowing the value of the smoothing parameter to vary. By attributing a low value to the parameter within the appropriate locality, a sharp turn in the data or an evident structural break can be absorbed by the trend, thereby allowing the residual sequence to maintain its normal behaviour.

A tapered sequence, based on successive repetitions of the ultimate seasonal cycle, can be added to the end of the data sequence, and a similar sequence, based on the first cycle, can be added before the start. However, such extrapolations tend to misrepresent the seasonal fluctuations by imposing gradual reductions in their amplitude. The amplitudes can be preserved by inserting a segment into the circular data sequence in which the seasonal pattern in the final year is transformed gradually into the pattern of the first year. This process can described as one of morphing the data, which is an allusion to a popular technique in computer graphics.

Let $s$ be the number of months or seasons in the year, and let the data be supplemented by a sequence of points, indexed by $j=0,1, \ldots, N s-1$ that correspond to an integral number of years. To avoid the use of subscripted indices, let the (detrended) sample points be denoted by $g[t] ; t=0,1, \ldots, T-1$. Then, the first year and the final year are replicated $N$ times in sequences in which the elements are defined, respectively, by

$$
g^{S}[j]=g[j \bmod s] \quad \text { and } \quad g^{F}[j]=g[T-s+(j \bmod s)] .
$$

A convex combination of these sequences with varying weights is given by

$$
\begin{aligned}
g^{E}[j] & =\lambda_{j} g^{F}[j]+(1-\lambda) g^{S}[j], \quad \text { with } \\
\lambda_{j} & =\frac{1}{2}\left\{\cos \left(\theta_{j}\right)+1\right\}, \quad \text { where } \quad \theta_{j}=\frac{\pi j}{N s} .
\end{aligned}
$$

The weights $\lambda_{j}$ are described by a half-cycle of a raised cosine function. The resulting sequence can be added to the end of the linear data sequence, which 


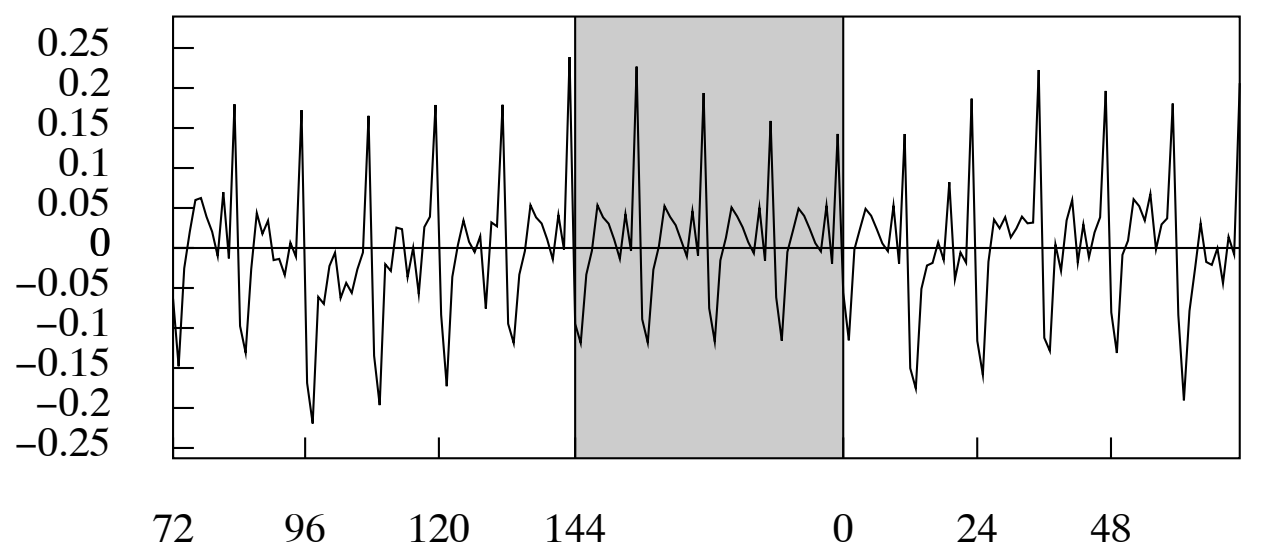

Figure 7. The residuals from a linear detrending of the sales data, with an interpolation of four years length inserted between the end and the beginning of the circularised sequence, marked by the shaded band.

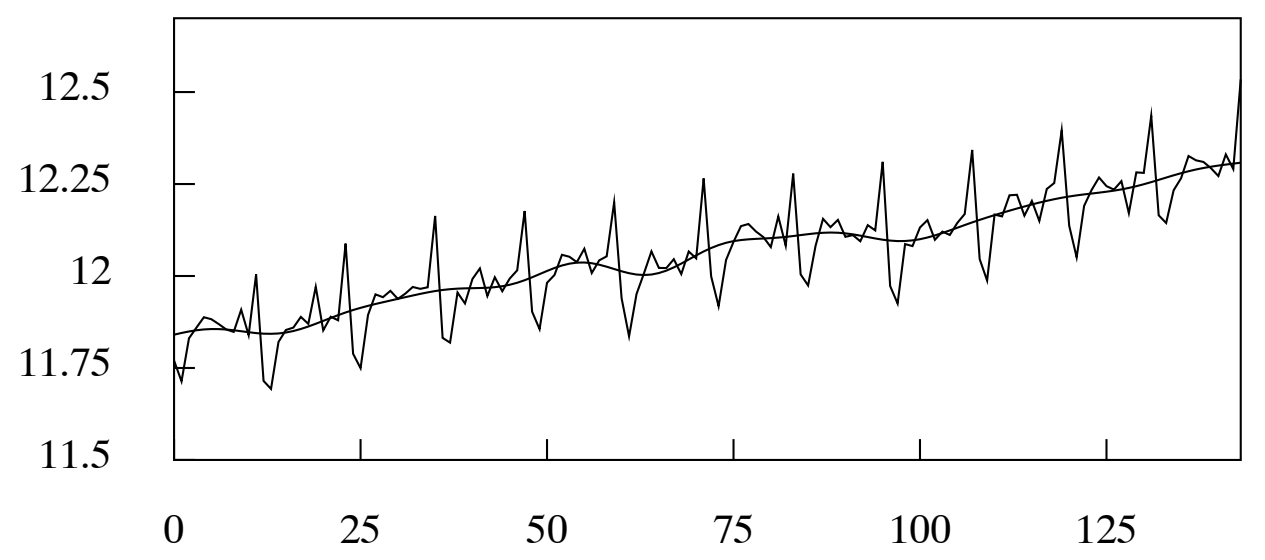

Figure 8. The logarithms of U.S. total retail sales from January 1953 to December 1964 with an interpolated trend-cycle function.

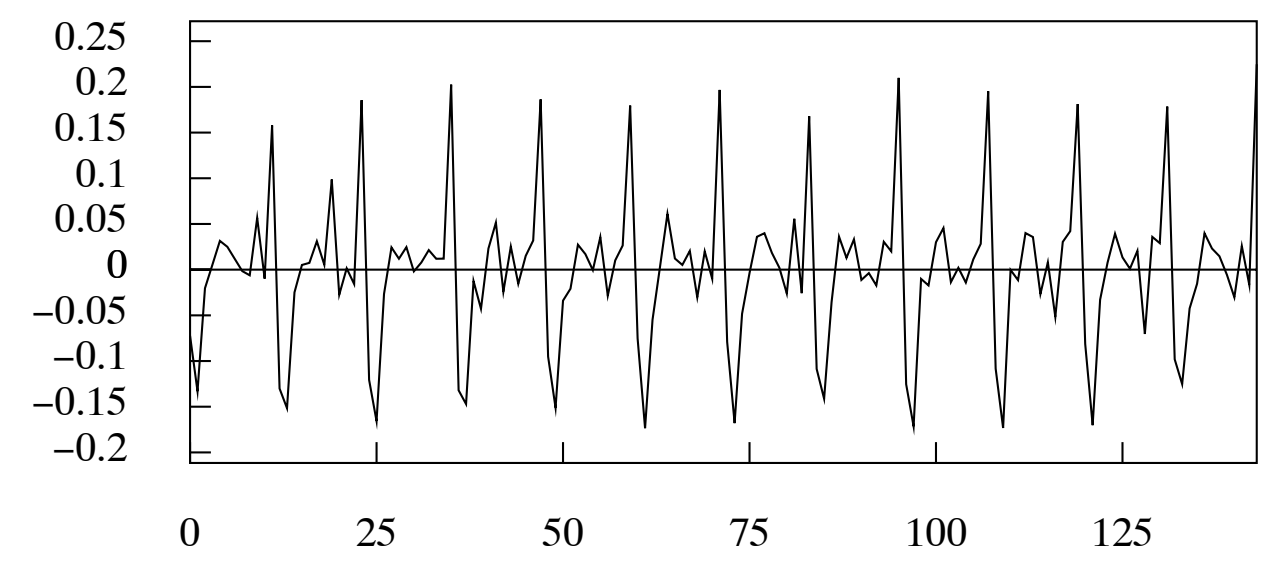

Figure 9. The sequence of residual deviations of the sales data from their trend, which may be regarded as the seasonal component. 


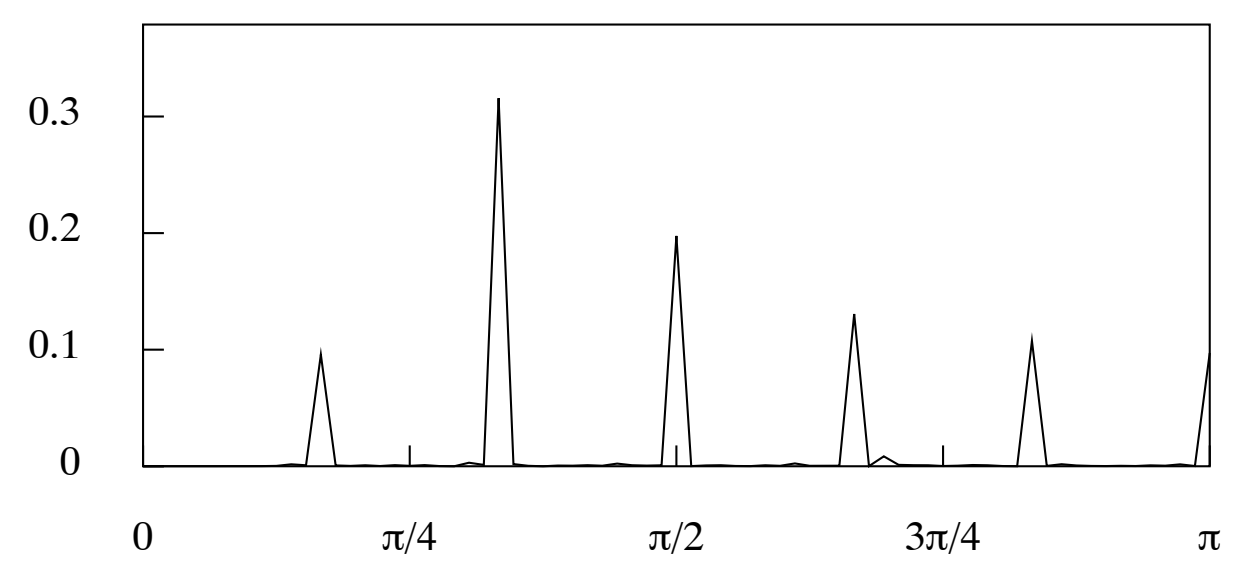

Figure 10. The periodogram of the residual deviations of the sales data from the trend depicted in Figure 8.

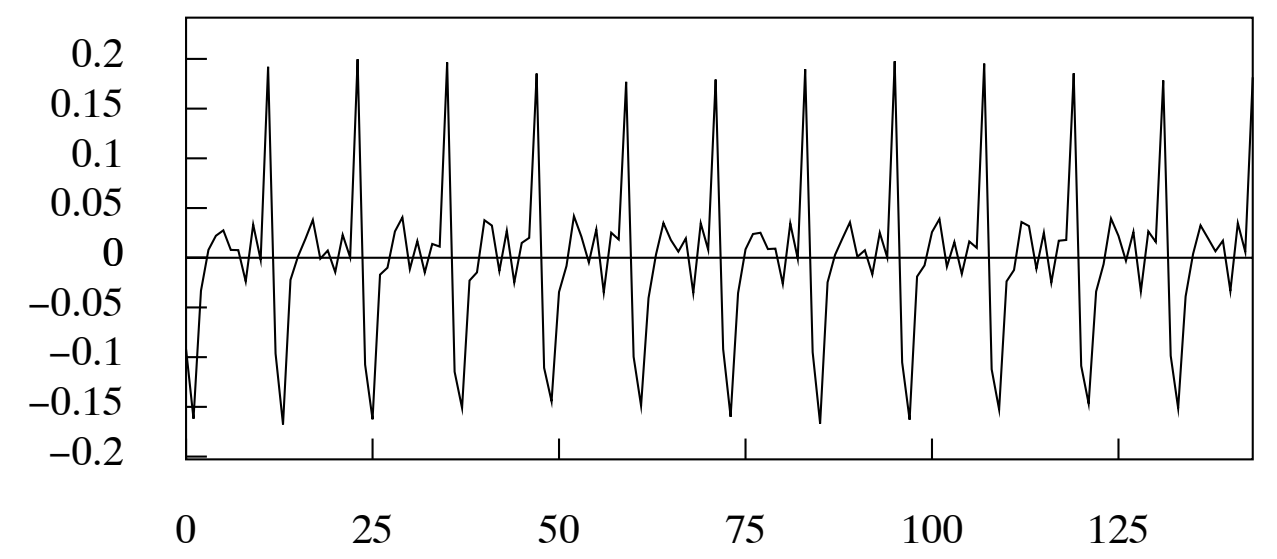

Figure 11. The seasonal component synthesised from the elements at the seasonal frequencies $\pi j / 6 ; j=1, \ldots, 6$ and from an element adjacent to the frequency of $2 \pi / 3$.

means that it will be interpolated between the finish and the start of the circular sequence.

The device of morphing is illustrated in a sequence of graphs relating to the logarithms of 144 monthly observations on retail sales in U.S. for the period from January 1953 to December 1964.

Figure 7 contains the residual deviations obtained by interpolating a linear trend through the logarithmic sales data, which are shown both in Figure 5 and in Figure 8. The residuals from the mid point of the sample to its end are displayed on the left of the figure. They are followed by a segment of artificial data, of four years duration, in which the pattern of the fluctuations of the final year of the data is gradually transformed into the pattern of the first year. This is followed by the residuals from the beginning of the sample to its mid point.

An inspection of the periodogram of Figure 6 of the residual sequence from the linear detrending shows that it possesses a low-frequency component falling in the interval $[0, \pi / 8]$. By removing this component, discarding the artificial elements and adding the remainder to the linear trend, the revised trend of Figure 8 is 


\section{D.S.G. POLLOCK: Econometric Signal Extraction}

generated.

By subtracting the revised trend from the data, a residual sequence is derived that is depicted in Figure 9. Its periodogram is displayed in Figure 10. Given that this periodogram contains almost nothing other than the prominent spikes at the seasonal frequency and its harmonics, it seems appropriate to regard this residual sequence as the seasonal component and to regard the revised trend - or trend-cycle - of Figure 8 as the seasonally adjusted data sequence.

A representation of the seasonal component can also be created from a synthesis of the six elements at the seasonal frequency and its harmonics and from the element that corresponds to the small spike in the periodogram at a frequency that is marginally in excess of $2 \pi / 3$. The resulting sequence is displayed in Figure 11. It closely resembles the residual sequence of Figure 9.

It follows that, by subtracting the synthesised seasonal sequence from the data, a sequence should be obtained that represents both the trend and the seasonally adjusted data. In fact, this sequence is affected by some of the roughness that is seen in seasonally adjusted data of Figure 5, which have been produced by a filter operating in the time domain. If it can be agreed that this roughness conveys no extra information, then it is indeed reasonable to regard the trend of Figure 8 as the most appropriate version of the seasonally adjusted data.

\section{References}

Bell, W., and Hillmer, S. (1988), A Matrix Approach to Likelihood Evaluation and Signal Extraction for ARIMA Component Time Series Models, Technical Report, U.S. Census Bureau.

Bloomfield, P., (1976), Fourier Analysis of Time Series: An Introduction, John Wiley and Sons, New York.

Box, G.E.P., and G.M. Jenkins, (1976), Time Series Analysis: Forecasting and Control, Revised Edition, Holden Day, San Francisco.

Caporello G., and A. Maravall, (2004), Program TSW, Revised Reference Manual, Servicio de Estudios, Banco de España.

Dagum, E.B., (1980), The X-11-ARIMA Seasonal Adjustment Method, Technical Report 12-564E, Statistics Canada, Ottawa.

Dagum, E.B., (1988), The X11-ARIMA/88 Seasonal Adjustment Method-Foundations and User's Manual, Time Series Research and Analysis Division, Statistics Canada Technical Report.

Findley, D.F., B.C. Monsell, W.R. Bell, M.C. Otto and B. Chen, (1998), New Capabilities and Methods of the X12-ARIMA Seasonal Adjustment Program, Journal of Business and Economic Statistics, 16, 127-152.

Findley, D.F., (2005), Some Recent Developments and Directions in Seasonal Adjustment, Journal of Official Statistics, 21, 343-365.

Findley, D.F., and D.K. Martin, (2006), Frequency Domain Analyses of SEATS and X-11/12-ARIMA Seasonal Adjustment Filters for Short and Moderate-Length Time Series, Journal of Official Statistics, 22, 1-34. 
Hillmer, S.C., and G.C. Tiao, (1982), An ARIMA-Model-Based Approach to Seasonal Adjustment, Journal of the American Statistical Association, 77, 63-70.

Hodrick, R.J., and E.C. Prescott, (1980), Postwar U.S. Business Cycles: An Empirical Investigation, Working Paper, Carnegie-Mellon University, Pittsburgh, Pennsylvania.

Hodrick, R.J., and E.C. Prescott, (1997), Postwar U.S. Business Cycles: An Empirical Investigation. Journal of Money, Credit and Banking, 29, 1-16.

Koopman S.J., A.C. Harvey, J.A. Doornik and N. Shephard, (2000), STAMP: Structural Time Series Analyser, Modeller and Predictor, Timberlake Consultants Press, London.

Ladiray, D., and B. Quenneville, (2001), Seasonal Adjustment with the X-11 Method, Springer Lecture Notes in Statistics 158, Springer Verlag, Berlin.

Leser, C.E.V., (1961), A Simple Method of Trend Construction, Journal of the Royal Statistical Society, Series B, 23, 91-107.

Monsell, B.C., J.A.D. Aston and S.J. Koopman, (2003), Toward X-13? Discussion paper of the U.S. Census Bureau, http://www . census.gov/ts/papers/jsm2003bcm.pdf

Monsell, B.C., (2009), An Update on the Development of X-13ARIMA-SEATS. In JJSM Proceedings, Business and Economic Statistics Section, Alexandria, VA: American Statistical Association.

Maravall, A., (1985), On Structural Time Series Models and the Characterization of Components, Journal of Business and Economic Statistics, 3, 350-355.

McElroy, T., and A. Sutcliffe, (2006), An Iterated Parametric Approach to Nonstationary Signal Extraction, Computational Statistics and Data Analysis, 50, 22062231.

Pollock, D.S.G., (1999), A Handbook of Time-Series Analysis, Signal Processing and Dynamics, Academic Press, London.

Pollock, D.S.G., (2002), A Review of TSW: The Windows Version of the TRAMOSEATS Program, Journal of Applied Econometrcs, 291-299.

Pollock, D.S.G., (2007), Wiener-Kolmogorov Filtering, Frequency-Selective Filtering and Polynomial Regression, Econometric Theory, 23, 71-83.

Shiskin, J., A.H. Young, and J.C. Musgrave, (1967), The X-11 Variant of the Census Method II Seasonal Adjustment, Technical Paper No. 15, Bureau of the Census, U.S. Department of Commerce.

Wiener, N., (1930), Generalised Harmonic Analysis, Acta Mathematica, 55, 117258. 